\title{
Using the Effect of Mechanical Stress on Doped Silicon as an Angular Movement Sensor for MOEMS/MEMS Micro Mirrors
}

\author{
D. Berko*, Y.S. Diamand \\ Dept of Physical Electronics, P.O. Box 39040, Tel Aviv 69978, Israel \\ *Corresponding author: danberko5@yahoo.com
}

Received January 23, 2014; Revised April 25, 2014; Accepted April 25, 2014

\begin{abstract}
The effect of elastic strain of moderate magnitude using high doped silicon substrate can change the conductivity of the substrate. The commonly used metal (strain) gage has a magnitude factor of between $2 \div 4$ while high doped silicon (strain) gage factor magnitude is between $150 \div 200$, thus improving the substrate sensitivity considerably. Using those physical attributes allow us to create a MOEMS sensor resolving accuracy issues and saving space in any future MOEMS device design. Those devices will be able to measure any mechanical movement connected to the high doped silicon substrate by converting the physical strain created from the movement stress to current/voltage change in the substrate device. The simplicity of the device is that the device could measure movement without any need to implement an outer sensor to it. By measuring the device's strain change it would "feel" the movement and convert it to an analog value, thus creating a strain gage built in the MOEMS device surface.
\end{abstract}

Keywords: mechanical stress, MOEMS/MEMS, angular movement sensor

Cite This Article: D. Berko, and Y.S. Diamand, "Using the Effect of Mechanical Stress on Doped Silicon as an Angular Movement Sensor for MOEMS/MEMS Micro Mirrors.” American Journal of Electrical and Electronic Engineering, vol. 2, no. 3 (2014): 88-91. doi: 10.12691/ajeee-2-3-5.

\section{Introduction}

Many of the MOEMS (Micro Optic Electric Mechanical System) development is micro mirrors devices that display an image or a video signal on a screen. Those devices usually use small sensors such as PSD (Position Sensitive Diode). Many of those devices encounter difficulties due to sometime pour image focus that derives from the difficulty to close the closed control loop between the mirror movements to the actual mirror position. Also those sensors are relatively quite big in the mirror device.

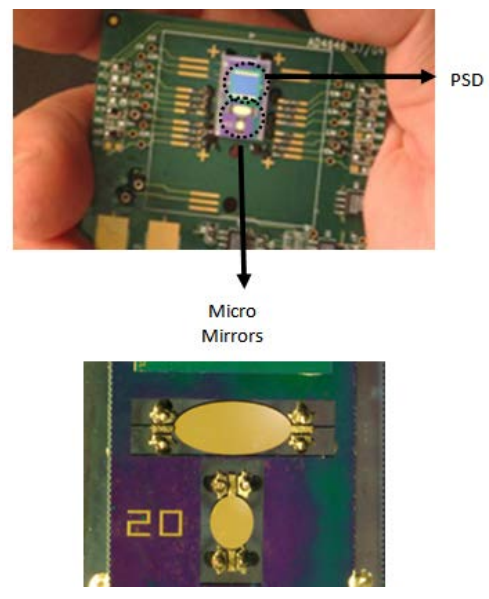

We wanted to create a "built in" sensor implemented in the mirror base rod substrate, thus simplifying the device and improving the sensor's reading and the image quality.

In order to measure angular movement of a micro mirror placed on a micro rod a mechanical stress conversion is needed. The stress resulting angular movement is torque stress, and in order to easily measure small strain change in the substrate we needed to convert it to a linear torsion or compression stress.

\section{Mechanical Stress Transformation of Torsion to Tensile and Compression}

Converting the torsion stresses to tensile and compression stresses was done by using new mechanical connection between the main beam of the mirror and its end connection. The new structures at the end connection were shaped as " $\mathrm{H}$ " for the micro mirror main beam. This solution is an originally development we invented and proved as very useful.

Getting a linear stresses distribution on a large surface made it easy to measure without limiting the micro mirror movement requirements. The constraint design of main mirror beam was very basic. The cube shaped connection gave no room for implanting any sensors or to create big surface to measure stresses. 


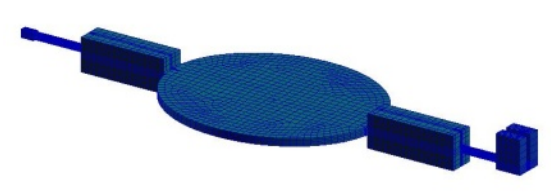

$K_{2}$

The first mirror beam design.

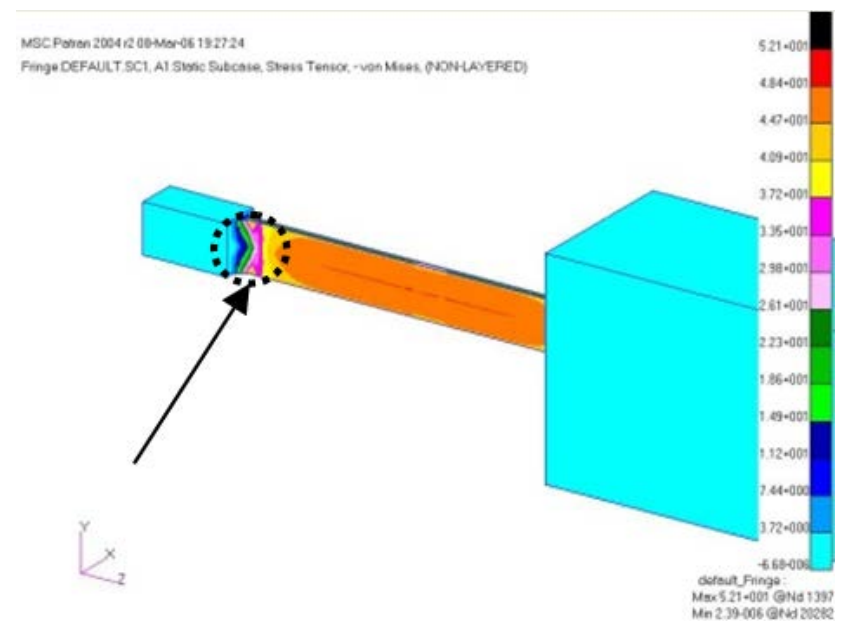

Finite elements analysis showing that the stress concentrates on a small and difficult area to monitor.

The new "H" design constraint.

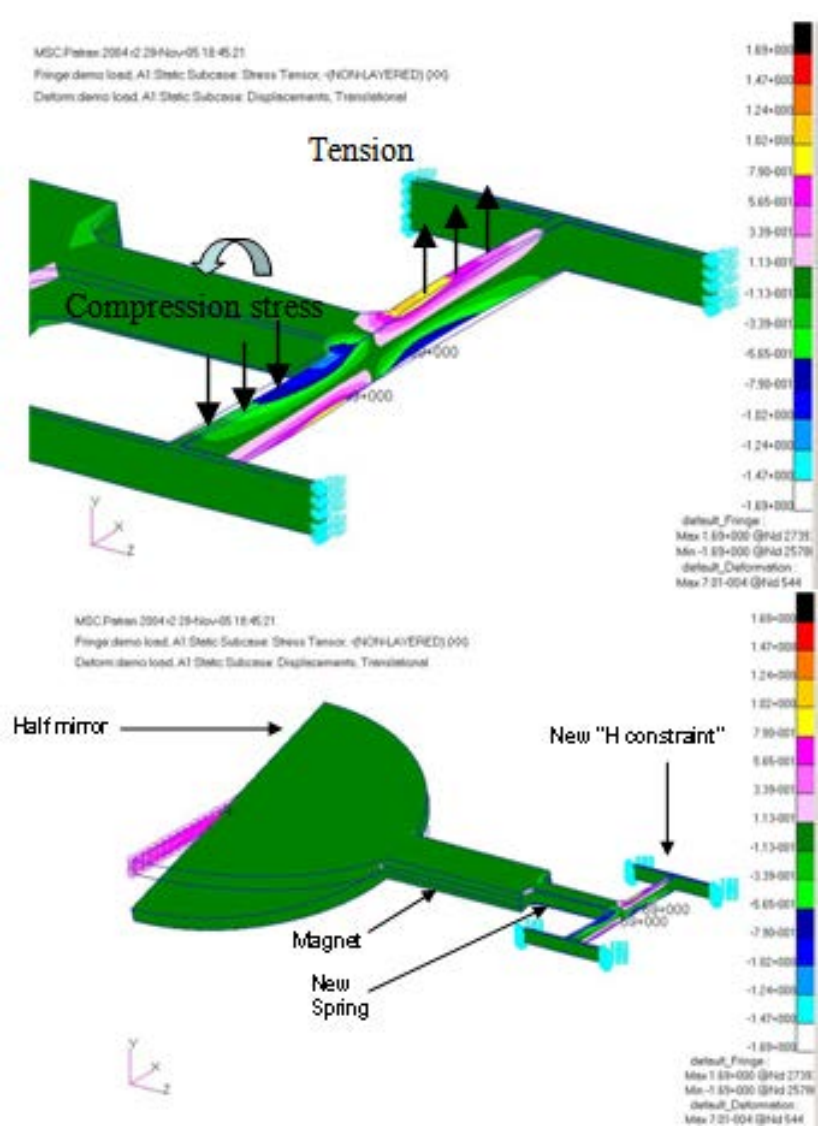

Finite elements analysis shows that changing the constraint design of the mirror main beam end connection to "H", converted the torsion stress to a uniformed compression and tensile stress area which is easier to monitor.

Mechanical analysis of the main beam mirror.

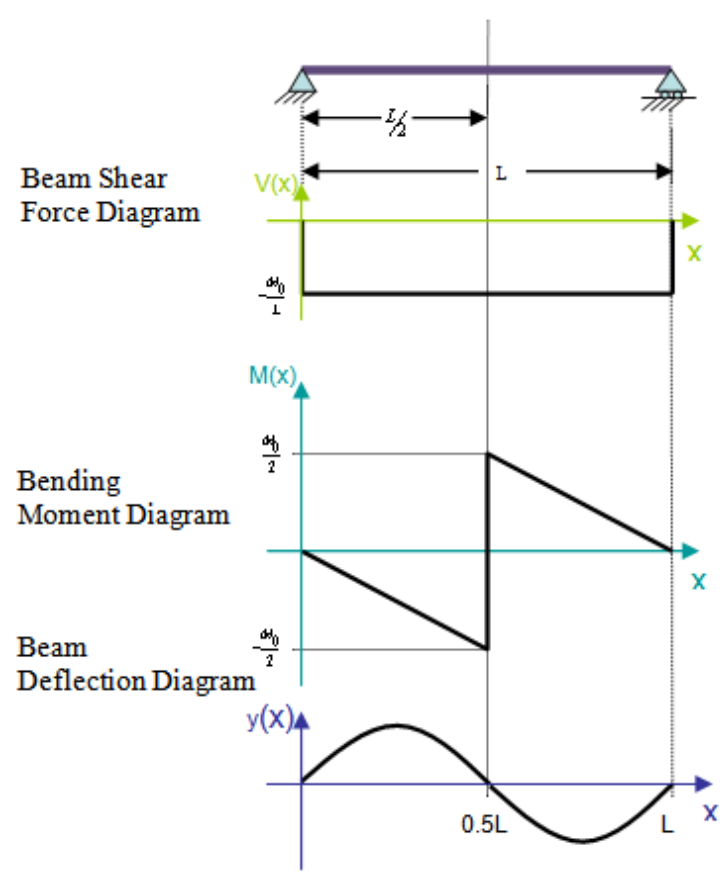

Maximum calculated moment:

$$
M(x)=-\frac{M_{0}}{L} \cdot x
$$

Maximum calculated beam stress:

$$
\sigma(x)=\frac{M \cdot C}{I}=\frac{\left(-\frac{M_{0}}{L} \cdot x\right) \cdot \frac{h}{2}}{\frac{b \cdot h^{3}}{12}}=-\frac{6 M_{0} \cdot x}{b \cdot h^{2} \cdot L}
$$

The strain-displacement relation is

$$
\varepsilon_{X X}=\frac{d u}{d x}
$$

In the case when the stain is $x$-dependent, the elongation of the gauge is:

$$
\begin{aligned}
& u=\int_{0}^{L / 2}-\frac{6 M_{0} \cdot x}{E \cdot b \cdot h^{2} \cdot L} \cdot d x=\left(-\frac{6 M_{0}}{E \cdot b \cdot h^{2} \cdot L} \cdot \frac{x^{2}}{2}\right)_{0}^{L / 2} \\
& u=-\frac{6 M_{0}}{E \cdot b \cdot h^{2} \cdot L} \cdot\left(\frac{L^{2}}{8}-0\right)=-\frac{3 M_{0} \cdot L}{4 E \cdot b \cdot h^{2}} \\
& u=\frac{3 \cdot 2 \cdot 697 \cdot 10^{-4} \cdot 0.78}{4 \cdot 17 \cdot 23 \cdot 10^{3} \cdot 10 \cdot 10^{-3} \cdot\left(70 \cdot 10^{-3}\right)^{2}}=-0.013 \mathrm{~mm}
\end{aligned}
$$

Maximum elongation of the beam is $0.013 \mathrm{~mm}$.

Calculation shows the same maximum elongation for the other half of the beam.

$$
\begin{aligned}
& u=\int_{L / 2}^{L}-\frac{6 M_{0}}{E \cdot b \cdot h^{2}}\left(\frac{x}{L}-1\right) \cdot d x=-\frac{6 M_{0}}{E \cdot b \cdot h^{2}} \cdot\left(\frac{x^{2}}{2 L}-x\right)_{L / 2}^{L} \\
& u=-\left[\frac{6 M_{0}}{E \cdot b \cdot h^{2}} \cdot\left(\frac{L}{2}-L\right)-\frac{6 M_{0}}{E \cdot b \cdot h^{2}} \cdot\left(\frac{L}{8}-\frac{L}{2}\right)\right]=-\frac{6 M_{0}}{E \cdot b \cdot h^{2}} \cdot\left(-\frac{L}{2}+\frac{3 L}{8}\right) \\
& u=\frac{6 M_{0}}{E \cdot b \cdot h^{2}} \cdot \frac{L}{8}=\frac{3 \cdot 2 \cdot 697 \cdot 10^{-4} \cdot 0.78}{4 \cdot 17 \cdot 23 \cdot 10^{3} \cdot 10 \cdot 10^{-3} \cdot\left(70 \cdot 10^{-3}\right)^{2}}=0.013 \mathrm{~mm}
\end{aligned}
$$


Both half beams are symmetric up to a sign difference.

The elongation is big enough to be effective and measurable by our sensor application.

The maximum strain of half a beam is:

$$
\varepsilon_{\max }=\frac{\Delta L_{\max }}{L / 2}=\frac{0.013}{0.78 / 2}=0.033
$$

\section{Electronic Design}

"H" surfaces will get opposite tensile and compression stresses. Those surfaces are the variable resistors of the Wheatstone bridge.

The surfaces are already doped thus have better sensitivity. Ref. resistors are doped surfaces with no stresses applied on them.
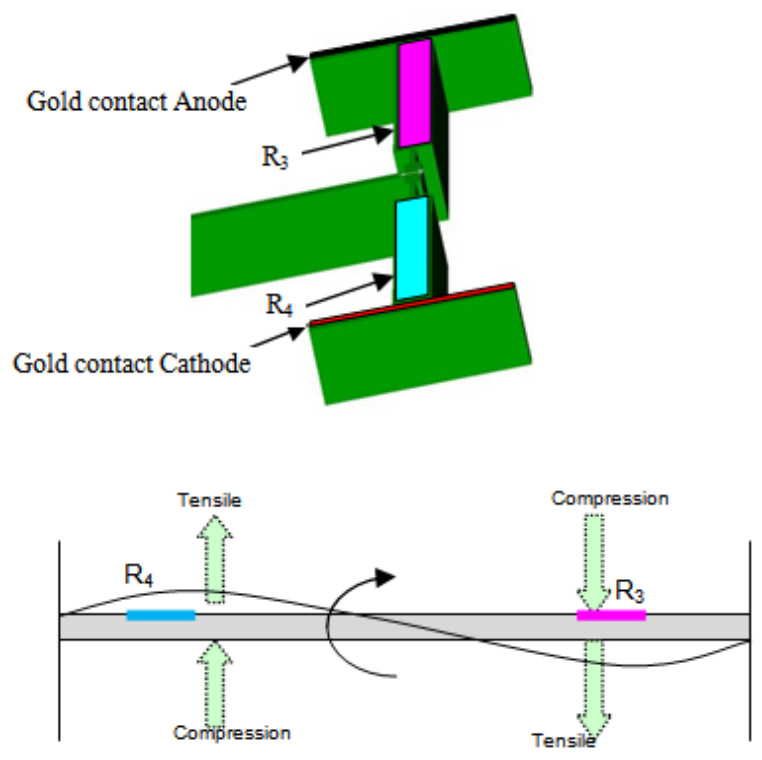

$$
\begin{aligned}
& \varepsilon=2 \cdot \frac{V_{0}}{V_{S}} \cdot \frac{1}{S}, \varepsilon=2 \cdot \frac{V_{0}}{V_{S}} \cdot \frac{1}{S} \\
& S F=S, G F=\frac{d \rho}{\varepsilon}>\varepsilon(1+v) \Rightarrow \frac{d R}{R} \approx \frac{d \rho}{\rho} \\
& G F=\frac{\Delta R / R}{\Delta L / L}=\frac{\Delta R / R}{\varepsilon}
\end{aligned}
$$

\section{High Doped Silicon as a Strain Gage}

The Electrical Principle of operation in order to create the built in sensor was first of all adding beams for the " $\mathrm{H}$ " constraint, thus adding big surfaces that are easier to implant MEMS sensors on. Secondly using high doped silicon to increase the conductivity of the substrate, thus enhancing the sensitivity of the built in sensor in the substrate. The gage factor of metal is between $2 \div 4$ while the high doped silicon gage factor is between $150 \div 200$. Thirdly using Wheatstone bridge to precisely measure the small changes in the resistance of the material due to applied stresses derived from angular movement. Another use of the Wheatstone bridge is to compensate temperature interferences and noises.

$$
R=\rho \frac{L}{A}=\frac{\rho}{h} \cdot \frac{L}{b}=R_{S} \cdot \frac{L}{b}, \frac{\rho}{h} \equiv R_{S}
$$

For high doped silicon $\rho=10^{-2} \div 10^{-1} \Omega \cdot \mathrm{cm}$

New added beam dimensions: $\mathrm{L}=780 \mu \mathrm{m}=0.078 \mathrm{~cm}$, but our elongation measurements were done for half beam so we shall use $\mathrm{L} / 2=0.039 \mathrm{~cm}$ in our calculations.

$$
\begin{aligned}
& \mathrm{b}=20 \mathrm{~mm}=2 \cdot 10^{-3} \mathrm{~cm}, \mathrm{~h}=70 \mathrm{~mm}=7 \cdot 10^{-3} \mathrm{~cm} \\
& R=\frac{\rho}{h} \cdot \frac{L}{b}=\frac{10^{-2}}{7 \cdot 10^{-3}} \cdot \frac{3.9 \cdot 10^{-2}}{2 \cdot 10^{-3}}=278.5 \Omega
\end{aligned}
$$

For gage factor 150 we get resistance delta of:

$$
\begin{aligned}
G F & =\frac{\Delta R / R}{\varepsilon} \\
\Delta R & =G F \cdot \varepsilon_{\max } \cdot R=150 \cdot 0.033 \cdot 278.5=1392 \Omega
\end{aligned}
$$

\section{Fabricating a Prototype Model}
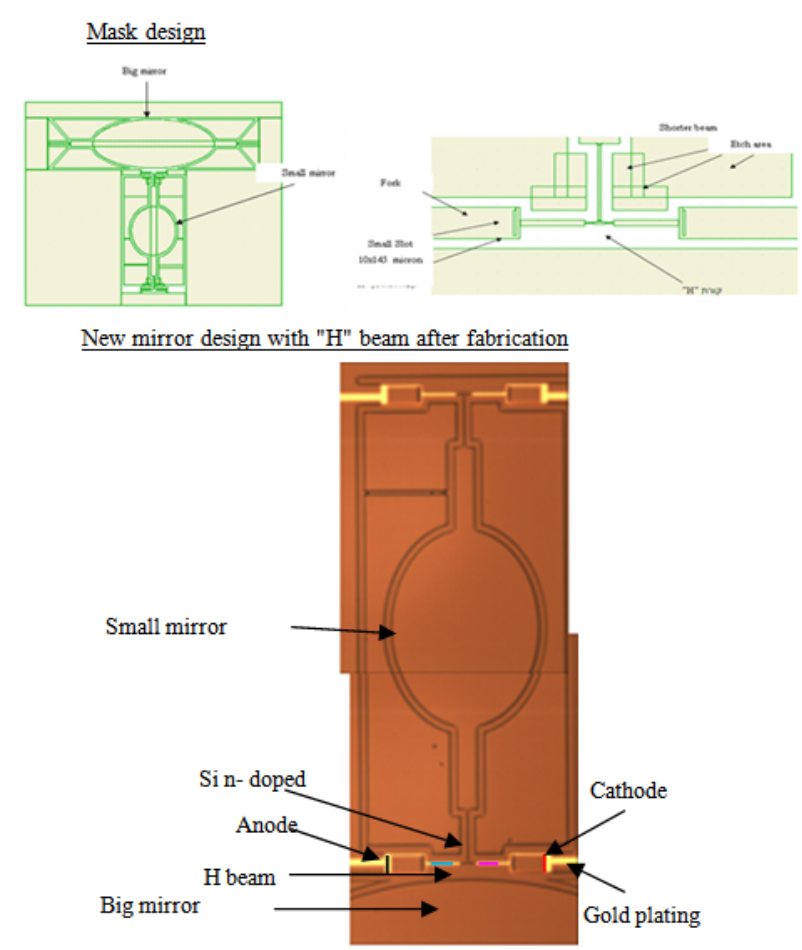

In this project the device was designed, masks were fabricated and manufacturing process flow was created for this specific model. Masks were fabricated in micro fabrication labs of Tel Aviv University, lithography, Dry Etch, Wet Etch and the production process was done in the labs. The process was unique and included complicated design and fabrication with Critical Dimensions as small as $10 \mu \mathrm{m}$ with depth of $70 \mu \mathrm{m}$.

The highly doped silicon substrate amplified the sensitivity of the measurement areas, thus enabling easier way to measure small angular movements of the micro 
mirror. The new device is simpler and takes smaller space then the old one (with the PSD).

\section{Conclusion}

The new design of built in sensor on silicon substrate as a strain gauge is new and innovative. It can change the method of MEMS measurements for micro mirrors and other applications. Those measurements are essential for closed loop control systems used in micro moving system applications. We hope this project will make it easier to develop devices for scanning images on small screens such as glasses, small projectors etc or any other MEMS designs it might have an effect on.

\section{References}

[1] Stephen A. Campbell, "The Science and Engineering of Microelectronic Fabrication", 2nd edition, Oxford, pp. 258-286.

[2] http://www.debiotech.com/debiotech.html

[3] Mohamed Gad-el-Hak, University of Notre Dame, "The MEMS Handbook", chapters 15-21.

[4] Danny Banks, "Microengineering, MEMS, and Interfacing: A Practical Guide (Mechanical Engineering), chapters 1-6. http://www.dbanks.demon.co.uk/ueng/index.html

[5] R. B. Darling / EE-527, EE-527: Micro Fabrication Photolithography, pp 1-31.

[6] Dr. A. Amin, "Piezoresistivity Theory and Application", Presentation for the IEEE-Ultrasonics, Ferroelectrics \& Frequency Control Society, pp 1-62 http://www.ieeeuffc.org/education/Piezoresistivity_files/frame.ht m\#slide0207.htm

[7] Adrian Watt, "Wheatstone Bridges Tutorial", Absorb Physics for A - Level.

[8] Walt Kester, "Analog Devices Seminar Sensor Signal Conditioning", Section 4: Strain, Force, Pressure, and Flow, pp
4.1-4.14.

http://search.analog.com/search/default.aspx?query=Walt+Kester \&local=en

[9] Dan Haronian, "Direct Integration (DI) of Solid State Stress Sensors with Integrated Displacement Sensing" Department of Interdisciplinary Studies, Faculty of Engineering, Tel-Aviv University, 0-7803-5194-0/99, 1999 IEEE, pp 88-93.

[10] http://robotics.eecs.berkeley.edu/ ronf/mfi.html/

[11] A. Eisinberg, A. Menciassi, S. Micera, D. Campolo, M.C. Carrozza, P. Dario, "PI Force Control of a Microgripper for Assembling Biomedical Micro devices".

[12] Maria Chiara Carrozza, Anna Eisinberg, Arianna Menciassi, Domenico Campolo, Silvestro Micera and Paolo Dario, "Towards a Force-Controlled Microgripper For Assembling Biomedical Micro devices", J. Micromech. Microeng. 10 (2000) 271- 276.

[13] Gisela Lin, Kristofer S. J. Pister, and Kenneth P. Roos, "Standard CMOS Piezoresistive Sensor to Quantify Heart Cell Contractile Forces", 0-7803-2985-6/96, 1996 IEEE, pp 150-155.

[14] Ingo Behrens, Lutz Doering and Erwin Peiner, "Piezoresistive cantilever as portable micro force calibration standard", Institut f"ur Halbleitertechnik, Technische Universit at Carolo-Wilhelmina zu Braunschweig, Germany, Journal of Micromechanics and Micro engineering, J. Micromech. Microeng. 13 (2003) pp S 171S 177.

[15] Romain Roduit, Pierre-Andr'e Besse, Member, IEEE, and JeanPaul Micallef "Flexible Angular Sensor", IEEE Transactions on Instrumentation and Measurement, VOL. 47, NO. 4, AUGUST 1998, pp 1020-1022.

[16] K.-U. Kirstein, Y. Li, M. Zimmermann, C. Vancura, T. Volden, W.H. Song, J. Lichtenberg, and A. Hierlemannn, "CantileverBased Biosensors in CMOS Technology", Physical Electronics Laboratory, ETH Zurich, Switzerlan, Proceedings of the Design, Automation and Test in Europe Conference and Exhibition (DATE’ 05) 1530-1591/05, IEEE.

[17] Joseph Edward Shigley, Charles R. Mischke, "Mechanical Engineering Design", 5th edition, McGraw Hill, pp 37-57, pp 91111, pp 725-763.

[18] Ralph Steiner, Christoph Maier, Michael Mayer, Sandra Bellekom, and Henry Baltes, "Influence of Mechanical Stress on the Offset Voltage of Hall Devices Operated with. 